\title{
Review of: "Impact of the COVID-19 lockdown on the epidemiology of maxillofacial trauma activity: a French multicentre comparative study"
}

\author{
Gianmarco Saponaro
}

Potential competing interests: The author(s) declared that no potential competing interests exist.

The article appears to be well written and it is interesting and representative of the French situation with regards of facial trauma panorama during the lockdown.

I would ask the author if they could somehow also give a comparisons of the age of the patients who referred to their centres in the considered periods of 2018-2019 and 2020, in order to see whether there was a variation in numbers and percentages adjusted per age.

Also it would be interesting to if there was a difference in the percentages of surgical indications A similar work was published as single centre in Italy: DOI:10.23736/S0026-4970.20.04446-5 It would be interesting to eventually make a comparison with the findings stated in the Italian paper.

Another comparison/discussion that I would find interesting is with this article DOI: 10.1016/j.amjsurg.2021.05.004 that states that no significant difference was noted for violent traumas, similarly to what the authors found in this very study.

In the discussion tha authors state "It appears that no study has yet been published on the variation in incidence of craniofacial trauma during the COVID-19 lockdown", this is actually not correct as paper DOI:10.23736/S0026-4970.20.04446-5 is a very similar study, although it is not multicenter, I would change to: "It appears that no multicenter study has yet been published for France on the variation in incidence of craniofacial trauma during the COVID-19 lockdown"

It think it can be published with minor fixes. 